\title{
Cavernomas pontinos extraventriculares. Parte II. Acesso pré-sigmoideo transpetroso a cavernoma pontino ventrolateral
}

Marcos Augusto Stávale Joaquim ${ }^{1}$, Gustavo Cartaxo Patriota², André de Macedo Bianco ${ }^{3}$

Instituto de Neurociências. São Paulo.

Serviço de Neurocirurgia Hospital 9 de Julho, São Paulo.

RESUMO

Objetivo: Descrever a via pré-sigmoidea ampliada para acesso a lesões localizadas na região ventrolateral da ponte, exemplificado com a apresentação de um caso de cavernoma pontino nessa localização. Conclusão: Esse acesso, amplamente revisto pela literatura, é útil para remoção cirúrgica de cavernomas pontinos ventrolaterais.

PALAVRAS-CHAVE

Cavernoma. Angioma cavernoso. Cirurgia do tronco cerebral.

\section{ABSTRACT}

Extraventricular pontine cavernomas. Part II. Pre-sigmoid, transpetrosal approach to ventrolateral pontine cavernomas

Objective: To describe the pre-sigmoid transpetrosal approach to the ventrolateral pontine region, exemplified by the presentation of a case of cavernoma in this localization. Conclusion: This approach is useful to remove pontine ventrolateral cavernomas.

\section{KEY WORDS}

Cavernoma. Cavernous angioma. Brain stem surgery.

\section{Introdução}

Os cavernomas do tronco encefálico podem provocar graves repercussões tanto pela hemorragia como pelo crescimento. $\mathrm{O}$ avanço da microcirurgia, da neuronavegação, da estereotaxia e da monitorização neurofisiológica intraoperatória possibilita a remoção dessas lesões com baixo risco cirúrgico, o que mudou a conduta atual. Lesões acessíveis devem ser removidas profilaticamente, desde que o planejamento prévio assim o permita. ${ }^{3,5,7,11}$ A via pré-sigmoidea, acesso amplamente revisto pela literatura, é útil para remoção cirúrgica de cavernomas pontinos de localização ventrolateral.

\section{Técnica cirúrgica}

Nesses casos, o paciente é operado em decúbito lateral, com planejamento estereotáxico ou por neuronavegação. Uma craniectomia retromastoidea é ampliada por meio de mastoidectomia e petrosectomia parcial, expondo-se o nervo facial. A cóclea e os canais semicirculares são removidos. O seio sigmoide é preservado. O orifício da veia de Santorini nos seios sigmodes é coagulado. O nervo facial é isolado. A abertura dural retromastoidea associa-se à abertura pré-sigmoidea. Assim, áreas de introdução do instrumental no compartimento cisternal tornam-se amplas.

1 Neurocirurgião do Instituto de Neurociências, São Paulo. Doutor em Medicina pela Faculdade de Medicina da Universidade de São Paulo (FMUSP).

2 Neurocirurgião do Instituto de Neurociências, São Paulo.

3 Neurocirurgião do Serviço de Neurocirurgia do Hospital 9 de Julho, São Paulo. 
As cisternas do ângulo pontocerebelar, pré-pontina e ambiens são adentradas. O trajeto do complexo nervoso vestibulococlear é seguido até o sulco bulbopontino. A artéria cerebelar ântero-inferior e uma alça descendente da artéria cerebelar superior são visualizadas. O nervo trigêmeo é isolado e o nervo abducente pode ser visualizado. ${ }^{5,12-14}$ A superfície lateral do tronco encefálico é visualizada no setor pontino, desde o sulco bulbopontino até o sulco pontomesencefálico. As veias locais são identificadas, mas muitas variações são possíveis. O trabalho é realizado no espaço petroclival médio. ${ }^{1,2,4,8-10,15-19}$

Seguindo-se o mapeamento, o ponto de intrusão na ponte é escolhido, poupando-se a topografia das fibras de maior competência funcional. Nessa região da ponte encontram-se fibras piramidais, núcleos pontinos e fibras transversas da ponte. Adjacente à lesão encontram-se fibras do nervo facial e abducente, o corpo trapezoide e o lemnisco medial. Também podem estar próximos o núcleo olivar superior e o núcleo do nervo trigêmeo. As fibras do pedúnculo cerebelar médio situam-se póstero-lateralmente.

Parte do cavernoma pode ser visível na superfície do tronco encefálico, ou um hematoma recente ou antigo (amarelado) pode eventualmente ser visto. Após intrusão no tronco encefálico, hematomas são delicadamente lavados e o cavernoma é, em geral, visualizado na sua parede. $\mathrm{O}$ ângulo de visão do microscópio deve permitir inspeção de toda cavidade. Apenas o cavernoma será removido e a gliose nos seus limites não é retirada ou lesionada. Preferencialmente, não se operam lesões na fase aguda das hemorragias, pois o tecido nervoso ao redor do coágulo é amolecido e sua lesão ou aspiração causará deficiências adicionais. Os angiomas venosos são preservados. Durante o procedimento é possível ocorrer a lesão da veia de Dandy. $\mathrm{O}$ fechamento minucioso impede fístulas liquóricas. A cavidade cirúrgica no tronco encefálico deve estar bem limpa antes do fechamento para não haver dúvida na ressonância pós-operatória. Nos casos de neoplasias, a ressonância deve ser realizada no primeiro dia após a cirurgia e áreas de captação refletem neoplasia macroscópica residual. Leva-se em conta que, após 48 a
72 horas, áreas de manipulação cirúrgicas e isquêmicas podem captar contraste e confundir a interpretação do exame. Nos casos de estudos pós-operatórios de cavernomas, a ressonância nuclear magnética poderá ser de difícil interpretação se houver sangue "recente", proveniente da cirurgia, no leito cirúrgico, dentro do tronco encefálico. Nesses casos, o controle a longo prazo revelará o resultado.

\section{Caso-exemplo}

PC, 21 anos, sexo feminino. Apresentou cefaleia hemicraniana e discreta síndrome de Millard-Gubler, com paresia facial homolateral à lesão e hemiparesia incompleta poupando a face contralateral. O quadro foi transitório e reverteu-se em semanas.

A ressonância nuclear magnética revelou um cavernoma hemorrágico pontino anterior à emergência do nervo trigêmeo e posterior ao trato piramidal e fibras transversas da ponte (Figuras 1 e 2). A cirurgia envolveu um acesso pré-sigmoideo ampliado anteriormente por petrosectomia, com exposição do nervo facial e, posteriormente, foi associada a técnica retrossigmoidea. $\mathrm{O}$ seio sigmoide foi preservado. A estereotaxia mostrou o acesso e o local de intrusão no tronco encefálico (Figura 3).
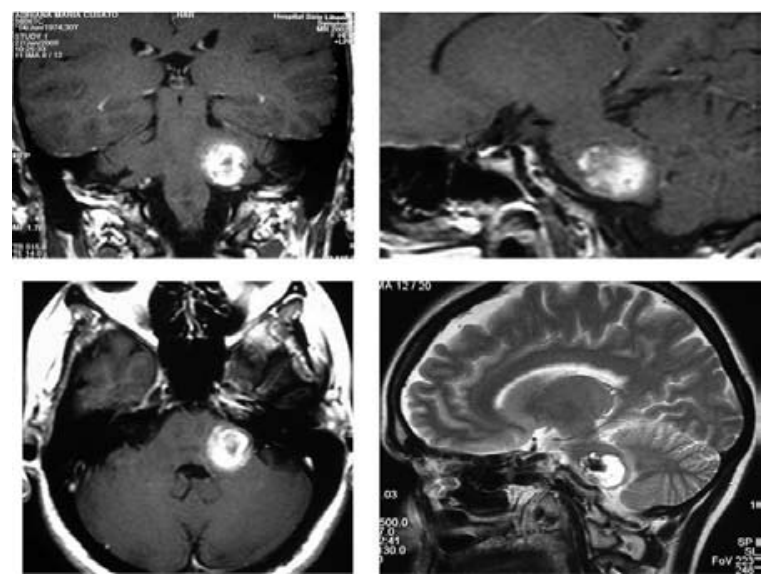

Figura 1-Ressonância magnética mostrando a topografia da lesão.
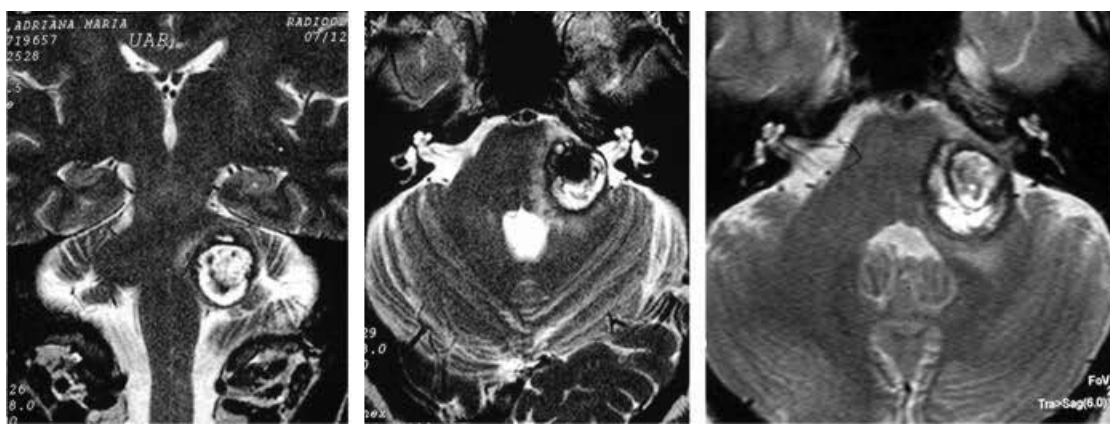

Figura 2 - Ressonância magnética, sequência T2.

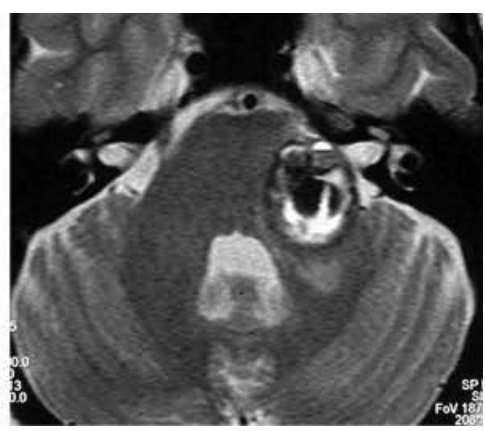




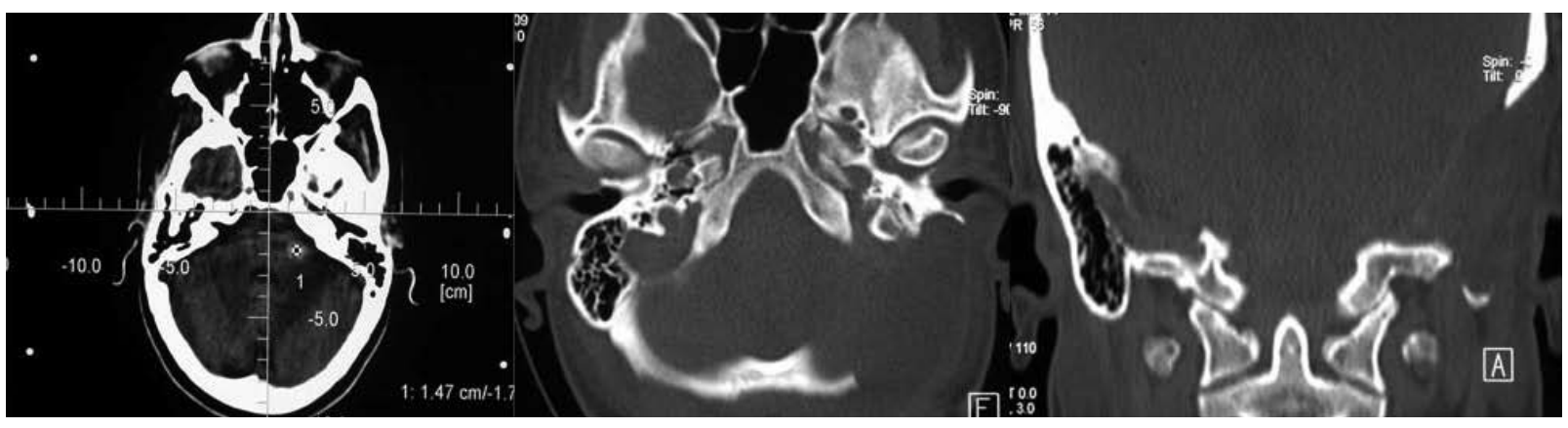

Figura 3 - Tomografia realizada para estereotaxia e tomografia mostrando osteotomia.

A monitorização neurofisiológica intraoperatória acusou frequentes disfunções e instabilidades elétricas do nervo facial. As funções piramidais apresentaram leve instabilidade por estímulo mecânico. A lesão foi removida preservando-se sua área gliótica adjacente (Figura 4). No período pós-operatório houve paralisia facial parcial que melhorou espontaneamente, mas não totalmente. Procedimento de cirurgia plástica foi necessário (cross-facing), tornando a disfunção imperceptível. Ocorreu anacusia definitiva homolateral, causada pela petrosectomia.
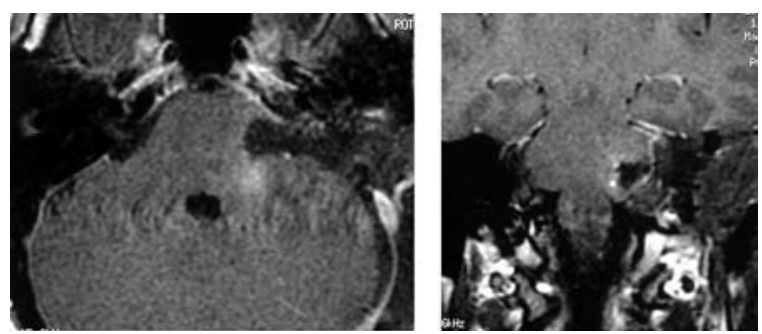

Figura 4-Ressonância magnética. Controle pós-operatório.

\section{Conclusão}

A face anterior da cisterna pré-pontina e do ângulo pontocerebelar, formada pelo clivo e porção medial da face posterior da pirâmide, é acessível por essa via. Do mesmo modo, suas faces posterior e medial, constituídas pela superfície ventral e ventrolateral do tronco encefálico, também são abordáveis. A remoção óssea adicional (osteotomias ampliadas) permite a visualização do conteúdo cisternal por vários ângulos e a escolha do melhor ponto de intrusão no tronco encefálico. $\mathrm{O}$ trabalho é realizado entre as estruturas neurovasculares locais, poupando-se a função. Portanto, lesões ventrolaterais da ponte podem ser abordadas por via pré-sigmoidea, que se associam ao conjunto de abordagens ao tronco encefálico.

\section{Referências}

1. Al-Mefty O, Fox JL, Smith RR. Petrosal approach for petroclival meningiomas. Neurosurgery. 1988;22:510-7.

2. Bricolo Ap, Turazzi S, Talacchi A, Cristofori L. Microsurgical removal of petroclival meningiomas: a report of 33 patients. Neurosurgery. 1992;31:813-82.

3. Drake CG. Surgical removal of arteriovenous malformation, from the brain stem and cerebellopontine angle. Neurosurgery. 1975;43:661-70.

4. Hakuba A, Nishimura S, Jang BJ. A combined retroauricular and preauricular transpetrosal-transtentorial approach to clivus meningiomas. Surg Neurol. 1988;30:108-16.

5. Haque R, Kellner CP, Solomon RA. Cavernous malformations of the brain stem. Clin Neurosurg. 2008;55:88-96.

6. Hardy DG, Rhoton AL. Microcirurgical relationships of the superior cerebelar artery and trigeminal nerve. J Neurosurg. 1983;59:63-105.

7. Hauch EF, Barnett SL, White JA, Samson D. Symptomatic brain stem cavernomas. Neurosurgery. 2009;64:61-71.

8. Kashimura $\mathrm{H}$, Inoue $\mathrm{T}$, Ogasawara $\mathrm{K}$, Ogawa A. Pontine cavernous angioma resected using the subtemporal, anterior transpetrosal approach determined using threedimensional anisotropy contrast imaging: technical case report. Neurosurgery. 2006;58(1 Suppl):ONS-E175.

9. Kawase T, Toya S, Shiobara R, Mine T. Transpetrosal approach for aneurysms of the lower basilar artery. J Neurosurg. 1985;63:857-61.

10. Kumabe T, Suzuki M, Yoshimoto T, Suzuki J. A case of cavernous angioma extend from the ventral part of the pons to the midbrain: subtemporal and trans-tentorial approach. No Shinkei Geka. 1988;16:1193-7.

11. Kupersmith MJ, Kalish H, Epstein F, Yu G, Berenstein A, Woo $\mathrm{H}$, et al. Natural history of brain stem cavernous malformations. Neurosurgery. 2001;48:47-53.

12. Martin RG, Grant JL, Peace D, Theiss C, Rhoton AL. Microsurgical relationships of the anterior inferior cerebelar artery and the facial-vestibulocochlear nerve complex. Neurosurgery. 1980;6:483-507.

13. Martinez AG, Oliveira E, Tedeschi H, Wen HT, Rhoton AL. Microsurgical anatomy of the brain stem. Oper Tech Neurosurg. 2000;3:80-6.

14. Matsuno M, Rhoton AL, Peace DP. Microcirurgical anatomy of the posterior fossa cisterns. Neurosurgery. 1988;23: 58-80.

15. Mayberg M, Symon L. Meningiomas of the clivus and apical petrous bone. Report of 35 cases. J Neurosurg. 1986;65:160-7. 
16. Sekhar LN, Estonillo R. Transtemporal approach to the skull base: an anatomical study. Neurosurgery.1986;19:799-808.

17. Sekhar LN, Schramm VL Jr, Jones NF. Subtemporal-preauricular infratemporal fossa approach to large lateral and posterior cranial base neoplasms. J Neurosurgery. 1987;67:488-99.

18. Sen CN, Sekhar LN. The subtemporal and preauricular infratemporal approach to intradural structures ventral to the brain stem. J Neurosurg. 1990; 73:345-54.

19. Tedeschi M, Rhoton AL. Lateral approaches to the petroclival region. Surg Neurol. 1994;41:180-216.
Original recebido em junho de 2008

Aceito para publicação em janeiro de 2009

Endereço para correspondência

Marcos Augusto Stávale Joaquim

Alameda Campinas, $1.360,16^{\circ}$ andar

01404-002 - São Paulo, SP, Brasil

E-mail:marcos.stavale@terra.com.br 